This item was submitted to Loughborough's Research Repository by the author.

Items in Figshare are protected by copyright, with all rights reserved, unless otherwise indicated.

\title{
Directed transport in a spatially periodic harmonic potential under periodic nonbiased forcing
}

PLEASE CITE THE PUBLISHED VERSION

http://dx.doi.org/10.1103/PhysRevE.79.026213

PUBLISHER

(C) American Physical Society

VERSION

VoR (Version of Record)

LICENCE

CC BY-NC-ND 4.0

\section{REPOSITORY RECORD}

Leoncini, Xavier, Anatoly Neishtadt, and Alexei Vasiliev. 2019. "Directed Transport in a Spatially Periodic Harmonic Potential Under Periodic Nonbiased Forcing”. figshare. https://hdl.handle.net/2134/15545. 
This item was submitted to Loughborough's Institutional Repository (https://dspace.lboro.ac.uk/) by the author and is made available under the following Creative Commons Licence conditions.

\section{creative
commons}

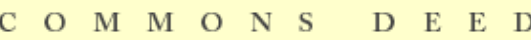

Attribution-NonCommercial-NoDerivs 2.5

You are free:

- to copy, distribute, display, and perform the work

Under the following conditions:

Attribution. You must attribute the work in the manner specified b the author or licensor.

Noncommercial. You may not use this work for commercial purposes.

No Derivative Works. You may not alter, transform, or build upon this work.

- For any reuse or distribution, you must make clear to others the license terms of this work.

- Any of these conditions can be waived if you get permission from the copyright holder.

Your fair use and other rights are in no way affected by the above.

This is a human-readable summary of the Leqal Code (the full license).

\section{Disclaimer 만}

For the full text of this licence, please go to: http://creativecommons.org/licenses/by-nc-nd/2.5/ 


\title{
Directed transport in a spatially periodic harmonic potential under periodic nonbiased forcing
}

\author{
Xavier Leoncini, ${ }^{1}$ Anatoly Neishtadt, ${ }^{2,3}$ and Alexei Vasiliev ${ }^{2, *}$ \\ ${ }^{1}$ Centre de Physique Théorique, Aix-Marseille Université, Luminy-case 907, F-13288 Marseille Cedex 09, France \\ ${ }^{2}$ Space Research Institute, Profsoyuznaya 84/32, Moscow 117997, Russia \\ ${ }^{3}$ Department of Mathematical Sciences, Loughborough University, Loughborough, LE11 3TU, United Kingdom
}

(Received 16 July 2008; revised manuscript received 5 January 2009; published 18 February 2009)

\begin{abstract}
Transport of a particle in a spatially periodic harmonic potential under the influence of a slowly timedependent unbiased periodic external force is studied. The equations of motion are the same as in the problem of a slowly forced nonlinear pendulum. Using methods of the adiabatic perturbation theory we show that for a periodic external force of a general kind the system demonstrates directed (ratchet) transport in the chaotic domain on very long time intervals and obtain a formula for the average velocity of this transport. Two cases are studied: The case of the external force of small amplitude, and the case of the external force with amplitude of order one. The obtained formulas can also be used in case of a nonharmonic periodic potential.
\end{abstract}

DOI: 10.1103/PhysRevE.79.026213

PACS number(s): 05.45.-a, 45.80.+r, 05.60.Cd

\section{INTRODUCTION}

In recent years, studies of transport phenomena in nonlinear systems have been attracting a growing interest. In particular, a large and constantly growing number of papers are devoted to dynamics in systems which allow for directed (on average) motion under unbiased external forces and are referred to as ratchet systems. Their intensive study was motivated by problems of motion of Brownian particles in spatially periodic potentials, unidirectional transport of molecular motors in biological systems, and recognition of "ratchet effects" in quantum physics (see Ref. [1] and references therein). Generally speaking, ratchet phenomena occur due to lack of symmetry in the spatially periodic potential and/or the external forcing. It is interesting, however, to understand microscopic mechanisms leading to these phenomena. A possible approach is to neglect dissipation and noise terms and arrive at a Hamiltonian system with deterministic forcing. Thus, one can make use of results obtained and methods developed in the theory of Hamiltonian chaos. Many papers studying chaotic transport in such Hamiltonian ratchets appeared in the past years (see, e.g., [2-8]). In particular, in [6] the ratchet current is estimated in the case when there are stability islands in the chaotic domain in the phase space of the system. The borders of such islands are "sticky" [9] and this stickiness together with desymmetrization of the islands are responsible for occurrence of the ratchet transport.

We consider the problem of motion of a particle in a periodic harmonic potential $U(q)=\omega_{0}^{2} \cos q$, where $q$ is the coordinate and $\omega_{0}=$ const, under the influence of unbiased time periodic external forcing. The equations are the same as in the paradigmatic model of a nonlinear pendulum under the action of external torque with zero time average. We study the case when the external forcing is a time-periodic function of large period of order $\varepsilon^{-1}, 0<\varepsilon \ll 1$, and use results and methods of the adiabatic perturbation theory. If $\varepsilon$ is small enough, there are no stability islands in the domain of cha-

\footnotetext{
*valex@iki.rssi.ru
}

otic dynamics (see [10]). Thus, the mechanism of ratchet transport in this system differs from one suggested in [6].

The main objective of this paper is to find a formula for the average velocity $V_{q}=\langle\dot{q}\rangle$ of a particle in the chaotic domain on very large time intervals. Chaos in the system is a result of multiple separatrix crossings due to slow variation of the external forcing. At each crossing the adiabatic invariant ("action" of the system) undergoes a quasirandom jump (see $[11,12]$ ). We show that these jumps result in effective mixing and uniform distribution of the action along a trajectory in the chaotic domain. On the other hand, direction and value of velocity depends on the immediate value of the action. Thus, to find the average velocity of transport on time intervals of order or larger than the mixing time, we find formulas for displacement in $q$ at a given value of the action and then integrate them over the interval of values of the action corresponding to the chaotic domain. We demonstrate that for an external force of general kind (i.e., with zero time average but lowered time symmetry, cf. [5]), there is directed transport in the system and obtain an analytic formula for the average velocity $V_{q}$ of this transport.

In Sec. II, we obtain the main equations in the case of external force of small amplitude and describe the diffusion of adiabatic invariant due to multiple separatrix crossings. This diffusion makes dynamics chaotic in a large domain of width of order 1. In Sec. III, we derive the formula for the average velocity of transport on very long time intervals (of order of typical diffusion time and larger) and check it numerically. In Sec. IV, we consider the case when the external forcing is not small, of order 1. Width of the chaotic domain in this situation is large, of order $\varepsilon^{-1}$. In this case chaos arises as a result of scattering of the adiabatic invariant on the resonance. Formula for the average velocity of transport in this case turns out to be much simpler than in the case of external forcing of small amplitude.

\section{MAIN EQUATIONS: DIFFUSION OF THE ADIABATIC INVARIANT}

We start with a basic equation describing a nonlinear pendulum under the influence of an external force $\widetilde{f}(t)$, 


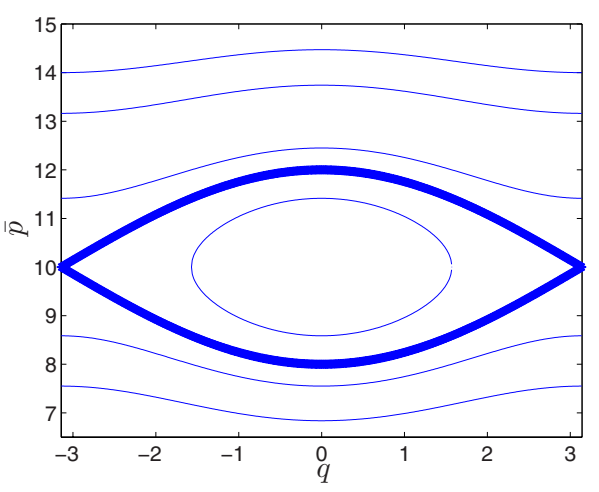

FIG. 1. (Color online) Phase trajectories of the system (3) with sample function $F(\tau)=A\left\{1+2 \exp \left[-\alpha(\sin \tau)^{2}\right]\right\}, A=10, \quad \alpha=8, \quad \tau$ $=\pi / 2, \omega_{0}=1$. The bold line is the separatrix.

$$
\ddot{q}+\omega_{0}^{2} \sin q=\tilde{f}(t) .
$$

We assume that $\tilde{f}(t)$ is small in amplitude periodic function of time of large period: $\widetilde{f}(t) \equiv \varepsilon f(\tau)=\varepsilon f(\tau+2 \pi)$, where $\tau$ $=\varepsilon t$. Moreover, we assume that $\tilde{f}(t)$ is a function with zero time average. This system is Hamiltonian, with timedependent Hamiltonian function

$$
H=\frac{p^{2}}{2}-\omega_{0}^{2} \cos q-\varepsilon f(\varepsilon t) q .
$$

We make a canonical transformation of variables $(p, q) \mapsto(\bar{p}, \bar{q})$ using generating function $W=[\bar{p}-F(\varepsilon t)] q$, where $F(\tau)=-\int f(\tau) d \tau$. Thus, $F(\tau)$ is a periodic function defined up to an additive constant, which we are free to choose. To make the following presentation more clear, we choose this constant in such a way that the minimal value of $F$ is $F_{\text {min }}>4 \omega_{0} / \pi$. Note that $\bar{q} \equiv q$. After this transformation, Hamiltonian of the system acquires the form (bars over $q$ are omitted)

$$
H=\frac{[\bar{p}-F(\tau)]^{2}}{2}-\omega_{0}^{2} \cos q .
$$

Phase portrait of the system at a frozen value of $\tau$ (we call it the unperturbed system) is shown in Fig. 1. The separatrix divides the phase space into the domains of direct rotations (above the upper branch of the separatrix), oscillations (between the separatrix branches), and reverse rotations (below the lower branch of the separatrix). Introduce the "action" I associated with a phase trajectory of the unperturbed system on this portrait. In the domains of rotation, $I$ equals an area between the trajectory, the lines $q=-\pi, q=\pi$, and the axis $\bar{p}=0$, divided by $2 \pi$; in the domain of oscillations, this is an area surrounded by the trajectory divided by $2 \pi$. It is known that $I$ is an adiabatic invariant of (3): Far from the separatrix its value is preserved along a phase trajectory with the accuracy of order $\varepsilon$ on long time intervals (see, e.g., [13]).

Location of the separatrix on the $(q, \bar{p})$ plane depends on the value of $F(\tau)$. As $\tau$ slowly varies, the separatrix slowly moves up and down, and phase points cross the separatrix and switch its regime of motion from direct rotations to reverse rotations and vice versa. Recall known results on varia- tion of the adiabatic invariant when a phase point crosses the separatrix. The area surrounded by the separatrix is constant, and hence, capture into the domain of oscillations is impossible in the first approximation (in the exact system, only a small measure of initial conditions correspond to phase trajectories that spend significant time in this domain; thus their influence on the transport is small). To be definite, consider the situation when the separatrix on the phase portrait slowly moves down. Thus, phase points cross the separatrix and change the regime of motion from reverse rotation to direct rotation. Let the action before the separatrix crossing at a distance of order 1 from the separatrix be $I=I_{-}$and let the action after the crossing (also at a distance of order 1 from the separatrix) be $I=I_{+}$. In the first approximation, we have $I_{+}=I_{-}+8 \omega_{0} / \pi$, i.e., the action increases by the value of the area enclosed by the separatrix divided by $2 \pi$ (see, e.g., $[14,15])$. We shall call this change in the action a "geometric jump." If the separatrix contour slowly moves up, and a phase point goes from the regime of direct rotation to the regime of reverse rotation, the corresponding value of the action decreases by the same value $8 \omega_{0} / \pi$. Thus, in this approximation, the picture of motion looks as follows. While a phase point is in the domain of reverse rotation, the value of $I$ along its trajectory stays constant, $I=I_{-}$. After transition to the domain of direct rotation, this value changes by the value of the geometric jump. The transition itself in this approximation occurs instantaneously. After the next separatrix crossing, the adiabatic invariant changes again by the value of the geometric jump, with the opposite sign, and returns to its initial value $I_{-}$. We call this approximation adiabatic.

In the next approximation, the value of action at the separatrix crossing undergoes a small additional jump. Consider for definiteness the case when the separatrix contour on the phase portrait moves down, and $I_{-}$and $I_{+}$are measured when it is in its uppermost and lowermost positions, accordingly. Results of $[11,12]$ imply the following formula for the jump in the adiabatic invariant:

$$
\begin{aligned}
2 \pi\left(I_{+}-I_{-}\right)= & 16 \omega_{0}+2 a(1-\xi) \varepsilon \Theta \ln (\varepsilon \Theta) \\
& +a \varepsilon \Theta \ln \frac{2 \pi(1-\xi)}{\Gamma^{2}(\xi)}-2 b \varepsilon \Theta(1-\xi),
\end{aligned}
$$

where $a=\omega_{0}^{-1}, b=\omega_{0}^{-1} \ln \left(32 \omega_{0}^{2}\right), \Theta=2 \pi F^{\prime}\left(\tau_{*}\right)$. Here $F^{\prime}$ is the $\tau$ derivative of $F, \tau_{*}$ is the value of $\tau$ at the separatrix crossing found in the adiabatic approximation, $\Gamma(\cdots)$ is the gamma function. Value $\xi$ is a so-called pseudophase of the separatrix crossing; it strongly depends on the initial conditions and can be considered as a random variable uniformly distributed on interval $(0,1)$ (see, e.g., [12]). Thus, value of the jump in the adiabatic invariant at the separatrix crossings has a quasirandom component of order $\varepsilon \ln \varepsilon$.

Accumulation of small quasirandom jumps due to multiple separatrix crossings produces diffusion of adiabatic invariant (see, e.g., [12]). On a period of $F(\tau)$ (after two separatrix crossings) the action changes by a value of order $\varepsilon \ln \varepsilon$. Hence, after $N \sim \varepsilon^{-2}(\ln \varepsilon)^{-2}$ separatrix crossings the adiabatic invariant varies by a value of order one. As a result, in time of order $t_{\text {diff }} \sim \varepsilon^{-3}(\ln \varepsilon)^{-2}$ the value of adiabatic invariant is distributed in all the range of values corresponding 

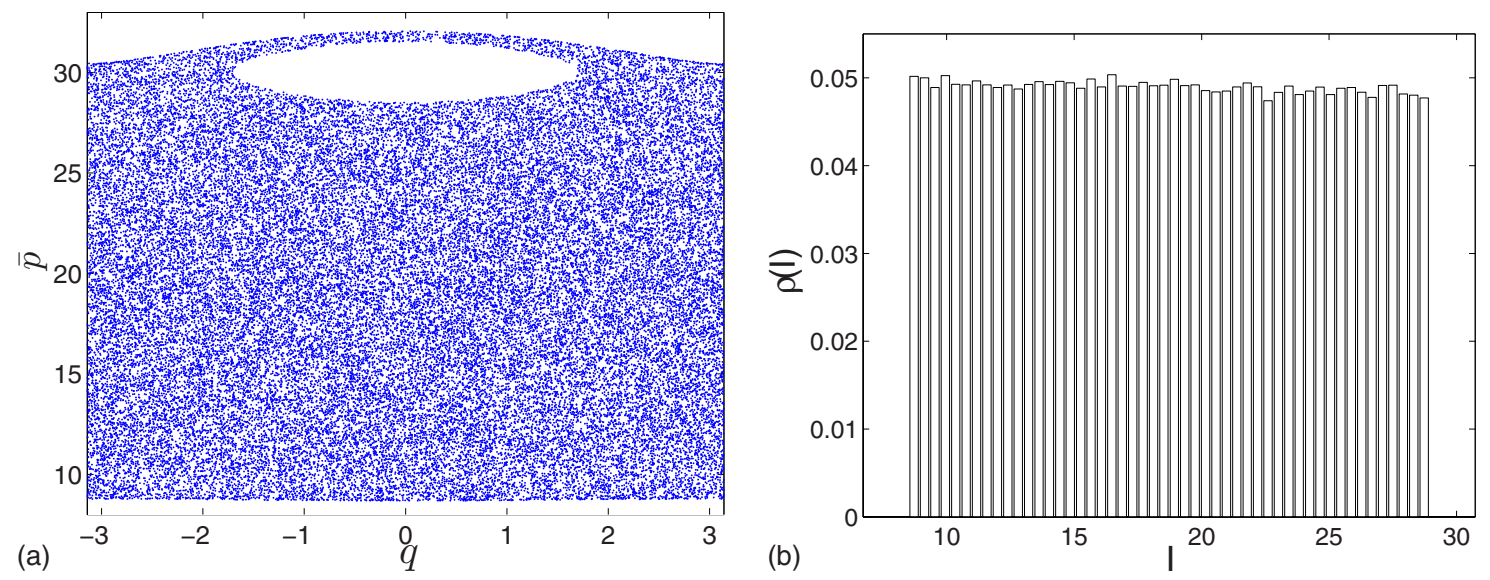

FIG. 2. (Color online) (a) Poincaré section at $\tau=0 \bmod 2 \pi$ of a long phase trajectory $\left(5 \times 10^{4}\right.$ dots). All the points are mapped onto the interval $q \in(-\pi, \pi) . F(\tau)=A\left\{1+2 \exp \left[-\alpha(\sin \tau)^{2}\right]\right\}$ with $A=10, \alpha=16, \varepsilon=0.005, \omega_{0}=1$. The empty region in the chaotic sea corresponds to phase points eternally locked in the domain of oscillations; they never enter the chaotic domain and do not participate in the transport. (b) Histogram of $I$ on the segment $\left(I_{\min }, I_{\max }-8 \omega_{0} / \pi\right)$ along the same phase trajectory.

to the domain where phase points cross the separatrix on the phase plane; its distribution is close to the uniform one. We have checked this fact numerically for a sample function $F(\tau)$ at various parameter values. Poincaré sections and distribution histograms of $I$ in all the cases look similar; see an example in Fig. 2.

\section{AVERAGE VELOCITY OF THE TRANSPORT}

Our aim is to find a formula for average velocity $V_{q}$ along a phase trajectory on time intervals of order $t_{\text {diff }}$ or larger. We first only take into consideration the geometric jumps, and afterwards, to obtain the final result, we take into account the mixing due to small quasirandom jumps. To simplify the consideration, assume that function $F(\tau)$ has one local minimum $F_{\min }$ and one local maximum $F_{\max }$ on the interval $(0,2 \pi)$. The main results are valid without this assumption.

Introduce $\tilde{I}$, defined in the domains of rotation as follows: it equals the area bordered by the trajectory, the line $\bar{p}$ $=F(\tau)$, and the lines $q=-\pi, q=\pi$, divided by $2 \pi$. Thus, $\tilde{I}$ $=|F(\tau)-I|$. Frequency of motion in the domains of rotation is $\omega(\widetilde{I})$, where $\omega(\widetilde{I})$ at $\widetilde{I}>4 \omega_{0} / \pi$ is the frequency of rotation of a standard nonlinear pendulum with Hamiltonian $H_{0}=p^{2} / 2$ $-\omega_{0}^{2} \cos q$, expressed in terms of its action variable $\widetilde{I}$. We do not need an explicit expression for function $\omega(\widetilde{I})$. From Hamiltonian (3) we find $\dot{q}=\bar{p}-F(\tau)$. Consider a phase trajectory of the system frozen at $\tau=\bar{\tau}$ in a domain of rotation. Let the value of action on this trajectory be $I=I_{0}$. Then the value of $\dot{q}$ averaged over a period $T$ of rotation equals $\int_{0}^{T}|\dot{q}| d t / T$ $=2 \pi / T=\omega\left[\left|F(\bar{\tau})-I_{0}\right|\right]$.

Now consider a long phase trajectory in the case of slowly varying $\tau$. Let on the interval $\left(\tau_{1}, \tau_{2}\right)$ a phase point of (3) be below the separatrix contour. In the adiabatic approximation, the value $I_{0}$ of the adiabatic invariant along its trajectory is preserved on this interval. Hence, at $\tau \in\left(\tau_{1}, \tau_{2}\right)$ we have

$$
2 \pi F(\tau)-2 \pi I_{0} \geqslant 8 \omega_{0},
$$

and the equality here takes place at $\tau=\tau_{1}$ and $\tau=\tau_{2}$. In the process of motion on this time interval, $q$ changes (in the main approximation) by a value

$$
\Delta q_{-}\left(I_{0}\right)=-\frac{1}{\varepsilon} \int_{\tau_{1}}^{\tau_{2}} \omega\left[F(\tau)-I_{0}\right] d \tau .
$$

On the interval $\left(\tau_{2}, \tau_{1}+2 \pi\right)$ the phase trajectory is above the separatrix contour, and the value of the adiabatic invariant equals $\hat{I}_{0}=I_{0}+8 \omega_{0} / \pi$ due to the geometric jump. On this interval we have

$$
2 \pi F(\tau)-2 \pi I_{0} \leqslant 8 \omega_{0}
$$

In the process of motion on this time interval, $q$ changes by a value

$$
\Delta q_{+}\left(I_{0}\right)=\frac{1}{\varepsilon} \int_{\tau_{2}}^{\tau_{1}+2 \pi} \omega\left[\left|F(\tau)-\hat{I}_{0}\right|\right] d \tau .
$$

Total displacement in $q$ on the interval $\left(\tau_{1}, \tau_{1}+2 \pi\right)$ equals $\Delta q\left(I_{0}\right)=\Delta q_{-}\left(I_{0}\right)+\Delta q_{+}\left(I_{0}\right)$, and the average velocity on this interval is $\varepsilon \Delta q\left(I_{0}\right) /(2 \pi)$.

Consider now the motion on a long enough time period

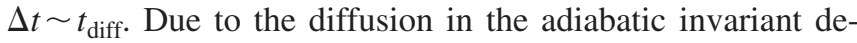
scribed above, in this time period values of $I_{0}$, defined as a value of $I$ when the phase point is below the separatrix contour, cover the interval $\left(I_{\min }, I_{\max }-8 \omega_{0} / \pi\right)$. Here $I_{\min }=F_{\min }$ $-4 \omega_{0} / \pi$ and $I_{\max }=F_{\max }+4 \omega_{0} / \pi$. Assuming that the distribution of $I$ on this interval is uniform, to find the average velocity, we integrate $\varepsilon \Delta q\left(I_{0}\right) /(2 \pi)$ over this interval. Integrating (5) over $I_{0}$ and changing the order of integration we find

$$
\begin{aligned}
\int_{I_{\min }}^{I_{\max }-8 \omega_{0} / \pi} \Delta q_{-} d I_{0} & =-\frac{1}{\varepsilon} \int_{0}^{2 \pi} d \tau \int_{I_{\min }}^{F(\tau)-4 \omega_{0} / \pi} \omega\left[F(\tau)-I_{0}\right] d I_{0} \\
& =-\frac{1}{\varepsilon} \int_{0}^{2 \pi} d \tau \int_{4 \omega_{0} / \pi}^{F(\tau)-I_{\min }} \omega(\eta) d \eta .
\end{aligned}
$$

Now we take into account the equality $\omega(\widetilde{I})=\partial H_{0}(\widetilde{I}) / \partial \widetilde{I}$ [recall that $H_{0}(\widetilde{I})$ is the Hamiltonian of a nonlinear pendulum as a function of its action variable] and obtain 

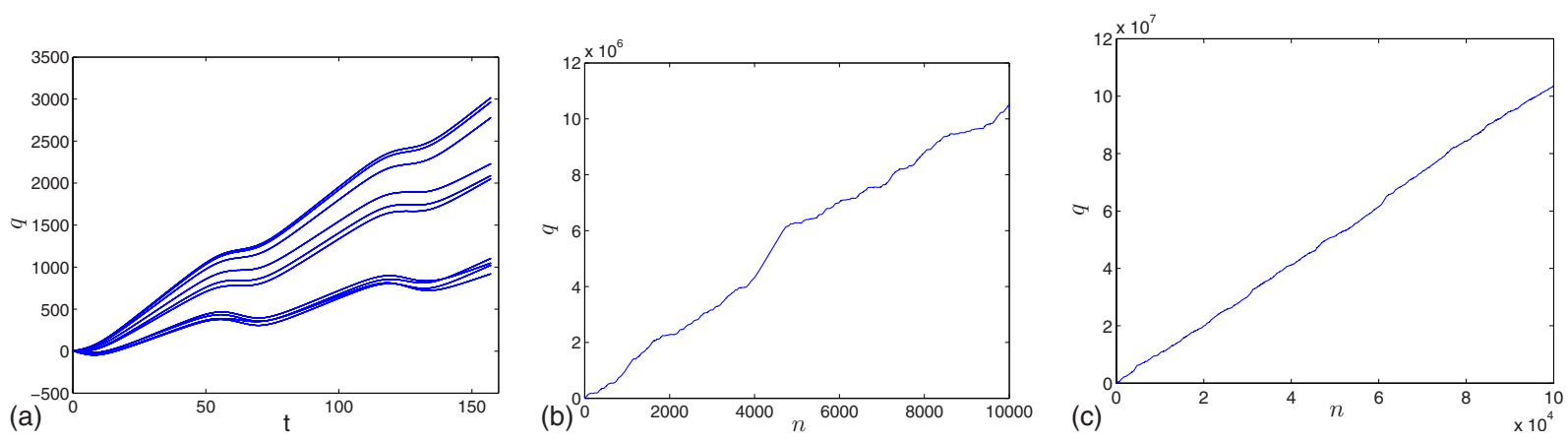

FIG. 3. (Color online) (a) $q$ against $t$ for 10 different initial conditions (comparatively short time interval), $\alpha=4, \varepsilon=0.05$. (b) $q$ against the number of periods of the external force for a sample trajectory $\left(10^{4}\right.$ periods), $\alpha=16, \varepsilon=0.05$. (c) $q$ against the number of periods of the external force for the same trajectory ( $10^{5}$ periods), $\alpha=16, \varepsilon=0.05$. Parameter $A=10$ in all cases.

$$
\begin{aligned}
-\int_{0}^{2 \pi} d \tau \int_{4 \omega_{0} / \pi}^{F(\tau)-I_{\min }} \omega(\eta) d \eta= & -\int_{0}^{2 \pi}\left\{H_{0}\left[F(\tau)-I_{\min }\right]\right. \\
& \left.-H_{0}^{s}\right\} d \tau
\end{aligned}
$$

where $H_{0}^{s}$ is the value of $H_{0}$ on the separatrix. Similarly, integrating (7) we obtain

$$
\int_{I_{\min }}^{I_{\max }-8 \omega_{0} / \pi} \Delta q_{+} d I_{0}=\frac{1}{\varepsilon} \int_{0}^{2 \pi}\left\{H_{0}\left[I_{\max }-F(\tau)\right]-H_{0}^{s}\right\} d \tau .
$$

Adding (8) and (9) and dividing by $2 \pi\left(F_{\max }-F_{\min }\right) / \varepsilon$ we find the expression for the average velocity $V_{q}$ of transport on long time intervals,

$$
\begin{aligned}
V_{q}= & \frac{1}{2 \pi\left(F_{\max }-F_{\min }\right)} \times \int_{0}^{2 \pi}\left\{H_{0}\left[I_{\max }-F(\tau)\right]\right. \\
& \left.-H_{0}\left[F(\tau)-I_{\min }\right]\right\} d \tau .
\end{aligned}
$$

In $(10), H_{0}(I)$ can be found as the inverse function to $\widetilde{I}(h)$, which defines action as a function of energy in domains of rotation of a nonlinear pendulum. For the latter function, the following formula holds (see, e.g., [16]):

$$
\tilde{I}(h)=\frac{4}{\pi} \omega_{0} \kappa \mathcal{E}(1 / \kappa), \quad \kappa \geqslant 1,
$$

where $\kappa^{2}=\left(1+h / \omega_{0}^{2}\right) / 2, \mathcal{E}(\cdots)$ is the complete elliptic integral of the second kind. If function $F(\tau)$ has several local extremes on the interval $(0,2 \pi), F_{\min }$ and $F_{\max }$ in (10) are the smallest and largest values of $F$, respectively.

It can be seen from (10) that $V_{q}$ is not zero for function $F(\tau)$ of general type, and hence there is the directed transport in the system. We checked this formula numerically for a sample function $F(\tau)=A\left\{1+2 \exp \left[-\alpha(\sin \tau)^{2}\right]\right\}, \quad \alpha>0$ at various values of parameters $\varepsilon$ and $\alpha$. Typical plots of $q$ against time $t$ are shown in Fig. 3.

The results of numerical checks of formula (10) are represented in Table I. To find numerical values of $V_{q}$ presented in the table, we integrated the system with Hamiltonian (3) on a long time interval $\Delta t=2 \pi \times 10^{6} / \varepsilon$ with a constant time step of $\pi / 100$ (fifth-order symplectic scheme [17]). Use of a symplectic scheme for long time simulations of Hamiltonian systems is necessary in order to ensure that creeping numerical error do not end up washing off the invariant tori bounding the chaotic domain. The table demonstrates satisfactory agreement between the formula and the numerics.

Finally, we note that formula (10) can be used also in the case of arbitrary (nonharmonic) spatially periodic time independent potential in place of the term $-\omega_{0}^{2} \cos q$ in (2) and (3). Of course, in this case function $H_{0}$ is different from the Hamiltonian of the nonlinear pendulum, but it always can be found, at least numerically.

\section{CASE OF NOT SMALL EXTERNAL FORCING}

In this section we study the case when the external forcing is not small. In this case, amplitude of function $\widetilde{f}(t)$ in $(1)$ is a value of order one, $\widetilde{f}(t) \equiv f(\tau)=f(\tau+2 \pi)$. The equations of motion are

$$
\dot{q}=p, \quad \dot{p}=-\omega_{0}^{2} \sin q+f(\tau), \quad \dot{\tau}=\varepsilon .
$$

One can see from the second equation, that magnitude of momentum $p$ can reach values of order $\varepsilon^{-1}$. Making the canonical transformation with generating function $W=[\bar{p}$ $\left.-\varepsilon^{-1} F(\varepsilon t)\right] q$, where $F(\tau)$ is defined in Sec. II, we obtain the Hamiltonian

$$
H=\frac{\left[\bar{p}-\varepsilon^{-1} F(\tau)\right]^{2}}{2}-\omega_{0}^{2} \cos q .
$$

Introduce $\tilde{p}=\varepsilon \bar{p}$ and rescaled time $\tilde{t}=\varepsilon^{-1} t$. We denote the derivative with respect to $\tilde{t}$ with prime and thus obtain

TABLE I. Numerically found values of $V_{q}$ corresponding to various values of parameters $\varepsilon, \alpha$ (four upper rows, $A=10, \omega_{0}=1$ ) and theoretical values $V_{q}^{\text {theor }}$ obtained according to (10) (the bottom row).

\begin{tabular}{llll}
\hline \hline & $\alpha=4$ & $\alpha=8$ & $\alpha=16$ \\
\hline$\varepsilon=0.1$ & 4.721 & 6.756 & 8.363 \\
$\varepsilon=0.05$ & 4.446 & 6.681 & 8.076 \\
$\varepsilon=0.01$ & 4.298 & 6.211 & 7.442 \\
$\varepsilon=0.005$ & 4.598 & 6.702 & 8.202 \\
$V_{q}^{\text {theor }}$ & 4.393 & 6.679 & 8.110 \\
\hline \hline
\end{tabular}



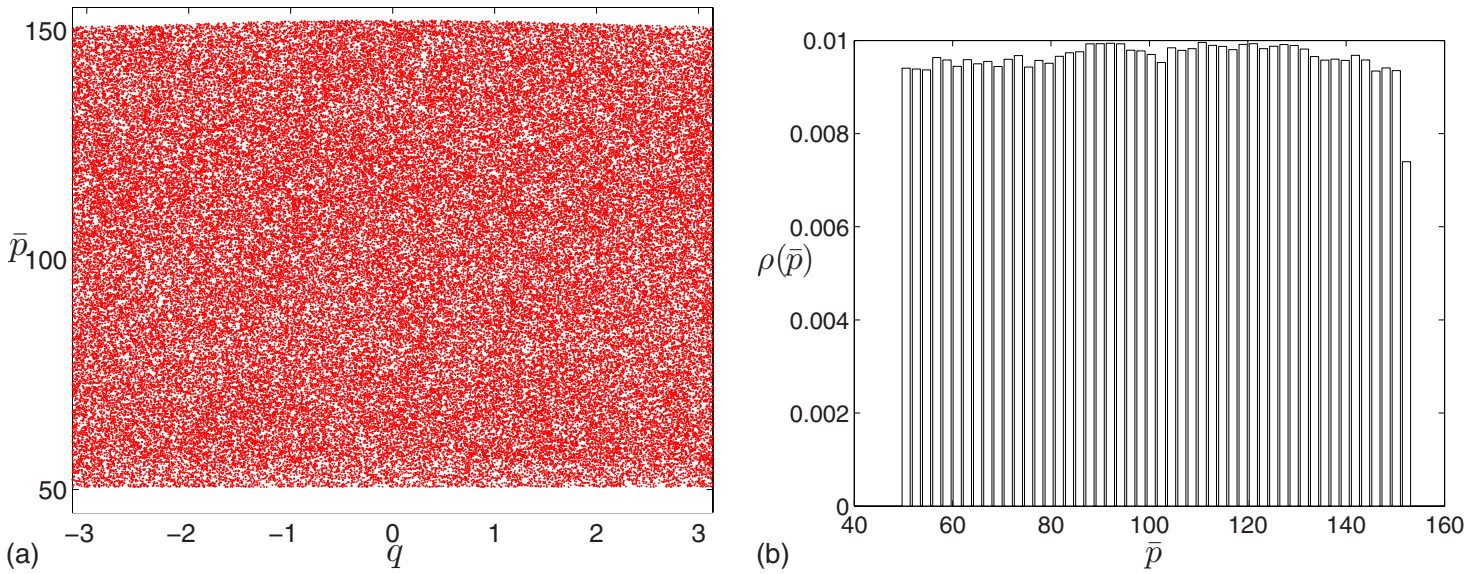

FIG. 4. (Color online) (a) Poincaré section at $\tau=0 \bmod 2 \pi$ of a long phase trajectory $\left(5 \times 10^{4}\right.$ dots) of system (13). All the points are mapped onto the interval $q \in(-\pi, \pi) . F(\tau)=A\left\{1+2 \exp \left[-\alpha(\sin \tau)^{2}\right]\right\}$ with $A=0.5, \alpha=4, \varepsilon=0.01, \omega_{0}=1$. (b) Histogram of $\bar{p}$ along the same phase trajectory.

$$
q^{\prime}=\tilde{p}-F(\tau), \quad \tilde{p}^{\prime}=-\varepsilon^{2} \omega_{0}^{2} \sin q, \quad \tau^{\prime}=\varepsilon^{2} .
$$

This is a system in a typical form for application of the averaging method. We average over fast variable $q$ and obtain the averaged system

$$
\tilde{p}^{\prime}=0, \quad \tau^{\prime}=\varepsilon^{2} .
$$

The averaged system describes the dynamics adequately everywhere in the phase space except for a small neighborhood of the resonance at $\tilde{p}-F(\tau)=0$, where the "fast" variable $q$ is not fast. When a phase trajectory of the averaged system crosses the resonance, value of the adiabatic invariant (in this case it coincides with $\tilde{p}$ ) undergoes a quasirandom jump of typical order $\sqrt{\varepsilon^{2}}=\varepsilon$ (see, e.g., $\left.[13,18]\right)$. Thus, the situation in this case is similar to one studied in Sec. II, but the typical value of a jump is of order $\varepsilon$, and, accordingly, the typical diffusion time is $t_{\text {diff }} \sim \varepsilon^{-3}$. Phase trajectories of the averaged system that cross the resonance correspond to values of $\tilde{p}$ belonging to the interval $\left(F_{\min }, F_{\max }\right)$. Therefore, the chaotic domain of the exact system is, in the main approximation, a strip $F_{\min } \leqslant \tilde{p} \leqslant F_{\max }$. Captures into the resonance followed by escapes from the resonance (see $[13,18]$ ) are also possible in this system. However, probability of capture is small, of order $\varepsilon$, and hence impact of these phenomena on the transport is small.

In Fig. 4 we represent a sample of Poincaré section of a long phase trajectory of (13) and the corresponding histogram of $\bar{p}$ for this trajectory. The plots show that the distribution of $\bar{p}$ is close to the uniform one.

As in Sec. III, one can find value $\Delta q$ on one period of perturbation, then average over the range of adiabatic invariant corresponding to the chaotic domain, and find the average velocity of transport in this case. Thus we find

$\Delta q=\int_{0}^{2 \pi / \varepsilon} p d t=\int_{0}^{2 \pi / \varepsilon}\left(\bar{p}-\frac{F(\tau)}{\varepsilon}\right) d t=\frac{1}{\varepsilon^{2}} \int_{0}^{2 \pi}[\tilde{p}-F(\tau)] d \tau$.

To find $V_{q}$, we must integrate this expression over $\tilde{p}$ from $F_{\min }$ to $F_{\max }$ (i.e., over the chaotic domain) and divide the result by $\left(F_{\max }-F_{\min }\right)$ and by the length of the period of the external forcing $2 \pi / \varepsilon$. Thus we obtain

$$
V_{q}=\frac{\varepsilon}{2 \pi\left(F_{\max }-F_{\min }\right)} \int_{F_{\min }}^{F_{\max }} \Delta q d \tilde{p} .
$$

Substituting $\Delta q$ from (16) and integrating, one straightforwardly obtains

$$
V_{q}=\frac{1}{4 \pi \varepsilon} \int_{0}^{2 \pi}\left[F_{\max }+F_{\min }-2 F(\tau)\right] d \tau .
$$

Another possibility to find $V_{q}$ is to use the already obtained formula (10). This way leads to the same result. When using (10), one should keep in mind that in the considered case $H_{0}$ in this formula is the Hamiltonian of the averaged system, i.e., $H_{0}=I^{2} / 2, I_{\max }=F_{\max }, I_{\min }=F_{\min }$, and that according to (13) we should set $\varepsilon^{-1} F$ instead of $F$ everywhere in the formula. Thus, (10) is much simplified, and we again arrive at formula (18). Factor $\varepsilon^{-1}$ is due to the fact that a phase point spends significant time moving at large velocities corresponding to $p \sim \varepsilon^{-1}$.

Note that formula (18) can be rewritten in a more elegant form as

$$
V_{q}=\frac{1}{\varepsilon}\left(\frac{F_{\max }+F_{\min }}{2}-\langle F(\tau)\rangle\right),
$$

where the angle brackets denote time average. The results of numerical checks of the formula are represented in Table II. As in Sec. IV, we integrated the system with Hamiltonian (13) on a long time interval $\Delta t=2 \pi \times 10^{6} / \varepsilon$.

Remarkably, formula (19) is valid for arbitrary smooth periodic potential (not necessarily harmonic) in place of the term $-\omega_{0}^{2} \cos q$ in (2) and (3). The potential may also depend periodically on time with the same period as that of the external force.

\section{SUMMARY}

To summarize, we have considered the phenomenon of the directed transport in a spatially periodic harmonic poten- 
TABLE II. Numerically found values of $\varepsilon V_{q}$ corresponding to various values of parameters $\varepsilon, \alpha$ in system (13) for $F(\tau)=A\{1$ $\left.+2 \exp \left[-\alpha(\sin \tau)^{2}\right]\right\}$ (four upper rows, $A=0.5, \omega_{0}=1$ ). In the bottom row theoretical values $\varepsilon V_{q}^{\text {theor }}$ obtained according to (19) are shown.

\begin{tabular}{llll}
\hline \hline & $\alpha=1$ & $\alpha=2$ & $\alpha=4$ \\
\hline$\varepsilon=0.1$ & 0.046 & 0.128 & 0.253 \\
$\varepsilon=0.05$ & 0.046 & 0.112 & 0.225 \\
$\varepsilon=0.01$ & 0.0353 & 0.1050 & 0.2044 \\
$\varepsilon=0.005$ & 0.0369 & 0.1081 & 0.1916 \\
$\varepsilon V_{q}^{\text {theor }}$ & 0.0389 & 0.1018 & 0.2006 \\
\hline \hline
\end{tabular}

tial adiabatically influenced by a periodic in time unbiased external force. We have shown that for the external force of a general kind the system exhibits directed transport on long time intervals. Direction and average velocity of the transport in the chaotic domain are independent of initial conditions and determined by properties of the external force. We studied two different cases: The case of small amplitude of the external force and the case, when this amplitude is a value of order one. We have obtained an approximate formula for average velocity of the transport and checked it numerically. The final formulas (10) and (19) are valid for any smooth periodic potential (not necessarily the harmonic one).

\section{ACKNOWLEDGMENTS}

The work was partially supported by the RFBR Grants No. 06-01-00117 and No. NSh 691.2008.1. A.V. thanks Centre de Physique Théorique in Luminy and Ricardo Lima for hospitality and numerous discussions.
[1] P. Reimann, Phys. Rep. 361, 57 (2002).

[2] P. Jung, J. G. Kissner, and P. Hänggi, Phys. Rev. Lett. 76, 3436 (1996).

[3] J. L. Mateos, Phys. Rev. Lett. 84, 258 (2000).

[4] O. Yevtushenko, S. Flach, and K. Richter, Phys. Rev. E 61, 7215 (2000).

[5] S. Flach, O. Yevtushenko, and Y. Zolotaryuk, Phys. Rev. Lett. 84, 2358 (2000).

[6] S. Denisov and S. Flach, Phys. Rev. E 64, 056236 (2001).

[7] S. Denisov, S. Flach, A. A. Ovchinnikov, O. Yevtushenko, and Y. Zolotaryuk, Phys. Rev. E 66, 041104 (2002).

[8] D. Hennig, L. Schimansky-Geier, and P. Hänggi, Eur. Phys. J. B 62, 493 (2008).

[9] G. M. Zaslavsky, Phys. Rep. 371, 461 (2002).
[10] A. I. Neishtadt and A. A. Vasiliev, Chaos 17, 043104 (2007).

[11] J. L. Tennyson, J. R. Cary, and D. F. Escande, Phys. Rev. Lett. 56, 2117 (1986).

[12] A. I. Neishtadt, Sov. J. Plasma Phys. 12, 568 (1986).

[13] V. I. Arnold, V. V. Kozlov, and A. I. Neishtadt, Mathematical Aspects of Classical and Celestial Mechanics (Encyclopedia of Mathematical Sciences 3) (Springer, Berlin, 2006).

[14] B. V. Chirikov, Sov. Phys. Dokl. 4, 390 (1959).

[15] A. I. Neishtadt, J. Appl. Math. Mech. 39, 594 (1975).

[16] R. Z. Sagdeev, D. A. Usikov, and G. M. Zaslavsky, Nonlinear Physics: From Pendulum to Turbulence and Chaos (Harwood Academic, Chur, Switzerland, 1992).

[17] R. I. McLachlan and P. Atela, Nonlinearity 5, 541 (1992).

[18] A. I. Neishtadt, Celest. Mech. Dyn. Astron. 65, 1 (1997). 